\title{
Survey of Elective Laryngeal Mask Airway Use in the Presence of Gastroe- sophageal Reflux Disease
}

\author{
Allison J. Lee* and Keith A. Candiotti \\ Department of Anesthesiology, Perioperative Medicine, and Pain Management, University of Miami Leonard M. Miller \\ School of Medicine, Miami, FL, USA
}

\begin{abstract}
Concern exists about the potential risks of pulmonary aspiration with the laryngeal mask airway (LMA) due to its inability to provide a tight seal at the larynx. The safety of LMA use in the presence of gastroesophageal reflux disease (GERD) is unclear, as GERD is presumed to increase the risk of aspiration under anesthesia. VA Anesthesiologists were surveyed regarding their practices in the setting of mild, moderately severe and severe symptoms of GERD. Approximately half responded that they would use a standard LMA in a patient with GERD as long as symptoms are mild. Nineteen percent would not use the LMA in any patient with a history of GERD.
\end{abstract}

Keywords: Laryngeal Mask Airway (LMA), Gastroesophageal Reflux Disease (GERD), Survey, Pulmonary Aspiration, General Anesthesia.

\section{INTRODUCTION}

Since it became commercially available in 1988, the Laryngeal Mask Airway (LMA) has become an extremely popular supraglottic airway device with over 200 million patient uses worldwide [1]. A drawback of the Classic LMA is that it does not provide a tight seal at the larynx and several case reports of aspiration have appeared in the literature [2]. Clinically detectable regurgitation with LMA use has been reported as 8.9 per 10,000 cases [3], while the incidence of aspiration, though unknown, has been suggested to be 2.3 per 10,000 cases based on data from prospective surveys [4]. The overall incidence of aspiration related to general anesthesia has been reported as 1.4-6.5 per 10,000 cases [4].

Gastroesophageal reflux disease (GERD) symptoms are frequently reported by surgical patients. The incidence of GERD in Western Europe and North America has been reported to be $10-20 \%$ [5], however, the true incidence of GERD in the general population is difficult to quantify, as large numbers of patients use over-the-counter medications to control their symptoms. The 2006 global consensus definition of GERD is: "a condition which develops when the reflux of stomach contents causes troublesome symptoms and/or complications". Population based studies concluded that mild symptoms occurring 2 or more times per week or severe symptoms occurring one or more times per week were considered troublesome [5].

It has been suggested that GERD should be considered a risk factor for perioperative aspiration [6]. GERD has not been specified by the manufacturer of the LMA as a contraindication to its use and they state that clinical judgment

*Address correspondence to this author at the Department of Anesthesiology, University of Miami Miller School of Medicine, Jackson Memorial Hospital, 1611 NW 12th Ave. (C-301), Miami, FL 33136, USA; Tel: (305) 585-7738; Fax: (305) 585-7012; E-mail: alee@med.miami.edu must be used to weigh the risk of regurgitation and aspiration against the potential benefit of establishing an airway [7].

In our experience, there appeared to be significant variations in practice among our colleagues, in the absence of evidence-based guidelines. An abstract from a 1998 survey of Australian Fellows of the Australian and New Zealand College of Anaesthetists reported that $57-73 \%$ of respondents would use the LMA in patients with a history of erosive esophagitis or hiatal hernia as long as the patient is symptom free [8]. No similar survey of US Anesthesiologists regarding LMA use was found in a MEDLINE search. With GERD so frequently encountered in surgical patients and with the huge popularity of the LMA, we hypothesized that a large number of anesthesiologists are willing to use a standard LMA in patients with a history of GERD, as long as the patients have mild or no symptoms. Anesthesiologists in the Veterans Affairs Healthcare System (VAHCS) were surveyed regarding the elective use of the LMA in patients who report a history of GERD, and what degree of symptoms affects their management choices.

\section{Materials and Methodology}

Following approval from the Miami VAHCS Human Studies Subcommittee, the link to a 14 question survey (Appendix 1) was emailed to 540 full-time Anesthesiologists in the VAHCS nationwide. Email addresses were derived from the VA payroll database. A reminder email was sent thirty days later, and follow-up phone calls were made to the VAHCS Anesthesiology Service Chiefs, to encourage participation.

To validate the reliability of the survey prior to distribution, the questions were sent to 21 practicing anesthesiologists, who completed the survey. The questions were resent two weeks later. A kappa statistic was used to analyze the reliability of each survey question. Reliability was noted to be fair to excellent on all questions. 
The survey data was tabulated as frequency and percentile of responses. The Chi-Square test was used to test for the association between responder characteristics and the use of LMAs. Analyses were performed with SAS 9.1 software.

\section{Results}

Of 540 emails sent, 239 responses were received (a 44\% response rate). No change in the pattern of responses was seen after the follow-up email or calls. The reported use of LMAs in the presence of GERD was independent of geographical region (Fig. 1), years of experience or type of practice.

Fifty-three percent of respondents reported being primarily employed in an academic setting, 3\% were primarily in private practice, $14 \%$ were in a mixed practice setting and $30 \%$ selected "Other" as their practice setting.

The largest subset of respondents (33.5\%) had 10-20 years experience. Almost all (97.4\%) use LMA's. The largest group (42.5\%) use LMA's for $10-25 \%$ of all general anesthetics. Most (91\%) never use LMA's for laparoscopic intra-abdominal surgery, however $39.5 \%$ use LMA's "sometimes" (or 25-49\% of the time) for surgeries in the lithotomy position. Forty-four percent were unsure whether an LMA with the capability of gastric decompression, such as the LMA ProSeal ${ }^{\mathrm{TM}}$ or LMA Supreme ${ }^{\mathrm{TM}}$ decreases the risk of pulmonary aspiration in patients with a history of GERD whereas $23.1 \%$ believed they would.

Approximately half of respondents reported that 25-50\% of their patients report GERD symptoms. Forty-nine per- cent said that moderately severe heartburn or greater would prevent them from placing a standard LMA whereas $18.7 \%$ reported they would avoid a standard LMA in anyone reporting a history of GERD, regardless of symptom severity (Table 1). For general endotracheal anesthesia for elective surgery, $21.9 \%$ said they would use acid suppression or gastrointestinal (GI) prophylaxis for all patients with a history of GERD whereas $36.3 \%$ would give GI prophylaxis only in the presence of moderately severe symptoms or greater. With respect to GI prophylaxis prior to LMA placement, the responses were mixed: $18.7 \%$ would give prophylaxis to all patients, regardless of symptom severity, $20 \%$ for mild symptoms and greater, $24.8 \%$ for moderate symptoms and greater, and $11.3 \%$ only for severe symptoms.

\section{Discussion}

Our objective was to determine practice standards among VAHCS Anesthesiologists regarding LMA use in patients reporting GERD. Almost all the anesthesiologists surveyed use LMAs and approximately half find $25-50 \%$ of their patients report GERD symptoms. Among respondents, there was no clear majority view, however, the responses indicate that almost half of respondents (49\%) would use an LMA in a patient with GERD as long as there are only mild symptoms, whereas about $19 \%$ of respondents would never use an LMA in any patient with GERD, regardless of symptom severity, presumably due to concerns regarding the risk of aspiration. Almost $17 \%$ reported that only severe GERD symptoms would prevent them from placing an LMA.

There is currently no gold standard for the diagnosis of GERD and an International Consensus Group agreed that the

In what region do you practice?

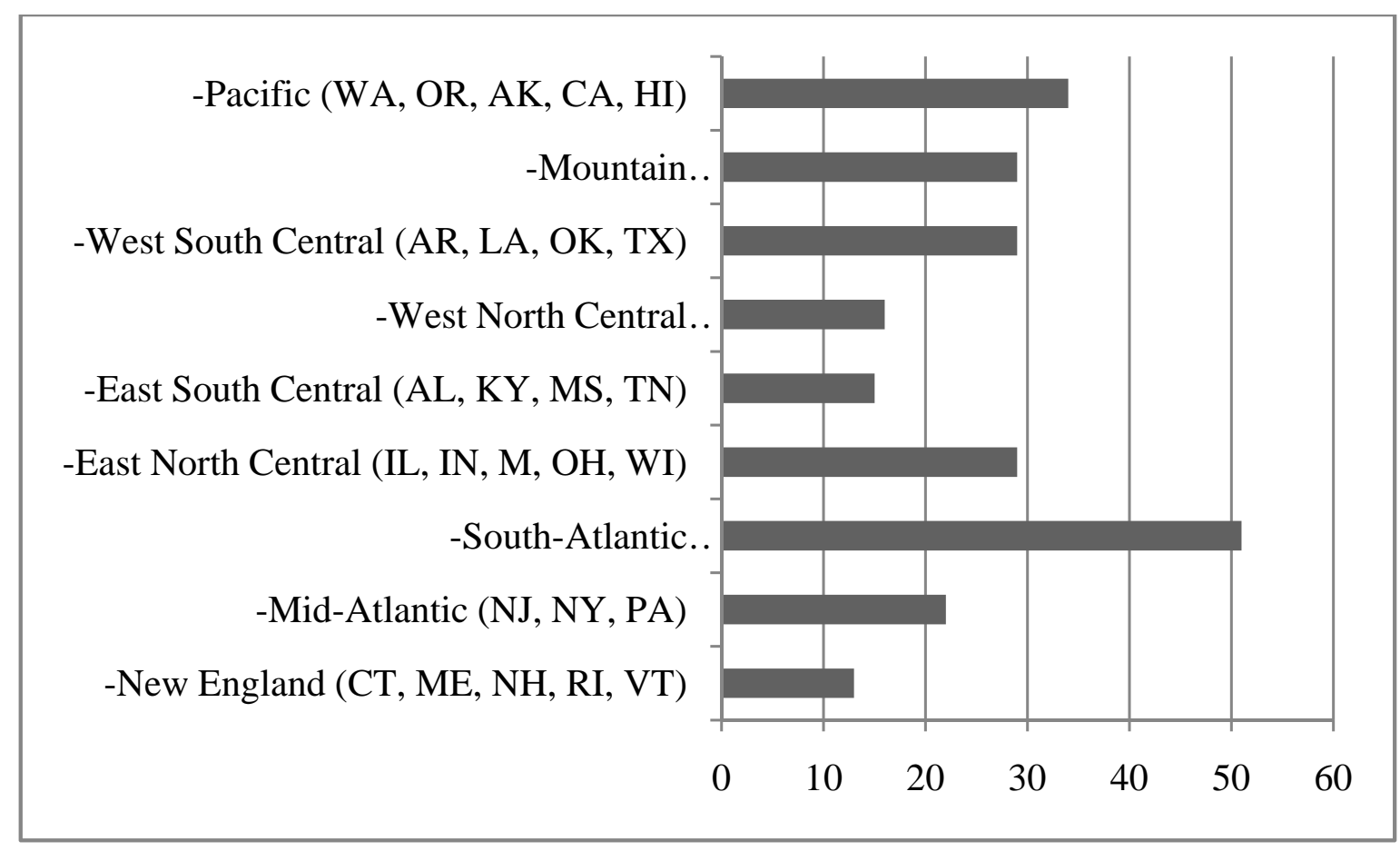

Fig. (1). Geographical region of respondents. 
typical reflux syndrome may be diagnosed on the basis of characteristic symptoms without diagnostic tests [5]. Only $5 \%$ of patients who report symptoms to their physician are referred for endoscopy in a given year [9]. Heartburn frequency and intensity have been found to have a moderate correlation with the severity of mucosal injury seen on endoscopy, however they may not always accurately predict the degree of mucosal injury in individual patients [5].

Table 1. Responses to Question: What Degree of Severity of GERD Symptoms would Prevent you from Placing a Standard LMA?

\begin{tabular}{|l|l|}
\hline & $\mathbf{n}(\boldsymbol{\%})$ \\
\hline \hline Severe heartburn symptoms & $39(16.6)$ \\
\hline Moderately severe heartburn Symptoms & $115(48.9)$ \\
\hline Mild heartburn symptoms & $37(15.7)$ \\
\hline $\begin{array}{l}\text { All patients with a history of GERD even if currently } \\
\text { asymptomatic }\end{array}$ & $44(18.7)$ \\
\hline
\end{tabular}

Although the incidence is unclear, a large proportion of patients who experience GERD symptoms are found to have endoscopy negative reflux disease (NERD) or functional dyspepsia [10]. It has been suggested that these patients may be abnormally sensitive to physiologic amounts of acid, or perhaps non-acid intra-esophageal stimuli trigger heartburn [10]. Conversely, elderly patients may have severe mucosal injury but no or decreased heartburn symptoms compared with younger patients $[5,11]$.

The International Consensus Group concluded that in clinical practice there is limited usefulness in arbitrary determinations of different categories of GERD severity based on frequency and duration and that the determination of how troublesome symptoms are should be patient-centered [5]. For these reasons, the questionnaire deliberately did not create specific definitions for categories of GERD severity, allowing respondents to report their typical practice choices based on the broad categories presented in the hypothetical clinical scenarios.

Episodes of reflux during general anesthesia with the LMA have been reported to be relatively frequent $[3,12$, 13]. Despite this, the reported rates of aspiration associated with LMA use are very low and approximate the incidence of aspiration prior to its introduction [13]. Most studies investigating regurgitation and the LMA exclude patients who have a history of GERD, therefore the specific risks in this setting are unknown. A prospective randomized study comparing 5 supraglottic airway devices (including the LMA) or an endotracheal tube found no difference in the frequency of regurgitation episodes during positive pressure ventilation [14].

A history of GERD and the severity of symptoms may not necessarily be related to the degree or frequency of reflux episodes under anesthesia. One prospective survey examined the use of the LMA in 2,359 patients [15]; of these, 17 were reported to have a hiatal hernia. No regurgitation was reported in any of those 17 patients. A small pro- spective study, investigating gastroesophageal reflux under anesthesia in 44 intubated patients, noted that a history of symptomatic esophagitis $(n=5)$ was predictive of reflux in only 2 patients whereas 4 patients with no history of reflux esophagitis developed reflux [16].

One limitation of this study is the relatively low response rate $(44 \%)$, which could have led to sampling bias. Some nonrespondents contacted, reported that they felt they were too busy to participate in the research survey or they felt they generally receive too many email surveys. A few physicians later reported technical issues accessing the survey. Another limitation in interpreting the data is that the practice of Anesthesiologists in the VAHCS may not necessarily reflect the practice of all US Anesthesiologists, particularly among private practitioners. The option for reporting LMA use regardless of GERD severity was not provided, based on the assumption that practitioners would be very unlikely to completely ignore GERD severity in their practice. However, this could have introduced bias in the results.

The LMA has been shown to have multiple advantages over endotracheal intubation, including increased speed of placement by anesthetists, improved hemodynamic stability at induction and emergence, minimal rise in intraocular pressure following insertion, reduced anesthetic requirements for airway tolerance, lower frequency of coughing during emergence, improved oxygen saturation during emergence, and lower incidence of sore throat in adults [17]. The risk of regurgitation and aspiration in patients with GERD symptoms who undergo elective surgery under general anesthesia with the LMA is unknown. Although consideration of these risks in patients with GERD is prudent, it is also desirable to avoid unnecessary tracheal intubation or use of rapid sequence induction for this indication. We believed it was important to address practitioners' choices for elective LMA use in this setting.

From this survey, it appears that a large percentage of VAHCS practitioners are willing to place an LMA in patients with mild GERD while others are firmly opposed. Individual practice choices cannot be strongly advocated or disputed, in the absence of clear evidence. Further studies are warranted, to examine the incidence and severity of reflux under general anesthesia in this patient population and to refine the risks of pulmonary aspiration in different sub-groups: symptomatic vs asymptomatic, treated vs untreated, as well as NERD and functional dyspepsia. These issues will presumably only continue to grow with the aging and increasing obesity of the American surgical population [18-21].

\section{CONCLUSION}

Practices among VAHCS anesthesiologists with respect to the elective use of the LMA in patients with a history of GERD appear to be varied. Almost half of respondents reported being comfortable using the LMA in patients with mild GERD symptoms, while $19 \%$ were firmly opposed to LMA use in any patient with a history of GERD.

\section{REFERENCES}

[1] Sung A, Kalstein A, Radhakrishan P, Yarmush J, Raoof S. Laryngeal mask airway: use and clinical applications. J Bronchol 2007; 14: $181-8$. 
[2] Keller C, Brimacombe J, Bittersohl J, Lirk P, von Goedecke A. Aspiration and the laryngeal mask airway: three cases and a review of the literature. Br J Anaesth 2004; 93: 579-82.

[3] McCrory CR, McShane AJ. Gastroesophageal reflux during spontaneous respiration with the laryngeal mask airway. Can J Anaesth 1999; 46: 268-70.

[4] Brimacombe J, Berry A. The incidence of aspiration associated with the laryngeal mask airway: a meta-analysis of published literature. J Clin Anesth 1995; 7: 297-305.

[5] Vakil N, van Zanten S, Kahrilas P, Dent J, Jones R, Global Consensus Group. The Montreal definition and classification of gastroesophageal reflux disease: a global evidence-based consensus. Am J Gastroenterol 2006; 101: 1900-20.

[6] Miller RD. Miller's Anesthesia. $6^{\text {th }}$ ed. USA: Churchill Livingstone 2004; p. 1860.

[7] Available from: http://www.lmana.com/indications.php. [Cited: 21st March, 2010].

[8] Crilly H, Mcleod K. Use of the laryngeal mask airway - a survey of Australian anaesthetic practice. Anaesth Intens Care 2000; 28: 224.

[9] Moayyedi P, Talley NJ, Fennerty MB, Vakil N. Can the clinical history distinguish between organic and functional dyspepsia? JAMA 2006; 295: 1566-76.

[10] Fass R, Tougas G. Functional heartburn: the stimulus, the pain, and the brain. Gut 2002; 51: 885-92.

[11] Johnson DA, Fennerty MB. Heartburn severity underestimates erosive esophagitis severity in elderly patients with gastroesophageal reflux disease. Gastroenterology 2004; 126: 660-4.
[12] Barker P, Langton JA, Murphy PJ, Rowbotham DJ. Regurgitation of gastric contents during general anaesthesia using the laryngeal mask airway. Br J Anaesth 1992; 69: 314-5.

[13] Brimacombe J, Berry A. Regurgitation and the laryngeal mask airway. Br J Anaesth 1993; 70: 381.

[14] Khazin V, Ezri T, Yishai R, et al. Gastroesophageal regurgitation during anesthesia and controlled ventilation with six airway devices. J Clin Anesth 2008; 20: 508-13.

[15] Verghese C, Smith TGC, Young E. Prospective survey of the use of the laryngeal mask airway in 2359 patients. Anaesthesia 1993; 48: 58-60.

[16] Illing L, Duncan PG, Yip R. Gastroesophageal reflux during anaesthesia. Can J Anesth 1992; 39(5): 466-70.

[17] Brimacombe J. The advantages of the LMA over the tracheal tube or facemask: a meta-analysis. Can J Anaesth 1995; 42: 1017-23.

[18] Fass R. The pathophysiological mechanisms of GERD in the obese patient. Dig Dis Sci 2008; 53: 2300-6.

[19] Available from: http://www.cdc.gov/obesity/data/trends.html. [Cited: 21st March, 2010].

[20] Kinsella K, Velkoff VUS. Census Bureau. An Aging World: 2001. Washington, DC: U.S. Government Printing Office 2001; series P95/01-1.

[21] Goulding MR, Rogers ME. Public health and aging: trends in aging United States and worldwide. MMWR: Morb Mortal Wkly Rep 2003: 52: 101-6.

(C) Lee and Candiotti; Licensee Bentham Open.

This is an open access article licensed under the terms of the Creative Commons Attribution Non-Commercial License (http://creativecommons.org/licenses/ by-nc/3.0/) which permits unrestricted, non-commercial use, distribution and reproduction in any medium, provided the work is properly cited. 